\title{
Recent physics results at BES
}

\author{
Zhiyong Wang* \\ IHEP, China \\ E-mail: wangzy@ihep.ac.cn
}

Using $58 \mathrm{M} \mathrm{J} / \psi, 14 \mathrm{M} \psi(2 S)$ and $33 \mathrm{pb}^{-1} \psi^{\prime \prime}$ events collected at BES-II detector, some charm physics results are obtained. In $J / \psi$ decay, we observed an unknown resonance $\mathrm{X}(1835)$ and study the controversial particle $\sigma$ and $\kappa$. In $\psi(2 S)$ decay, we test the "12\% rule" via many decay modes and find many new $\chi_{c J}$ decay channels. In $\psi^{\prime \prime}$ decay, we observed the non-D $\bar{D}$ decay via $\psi^{\prime \prime} \rightarrow \pi^{+} \pi^{-} J / \psi$ and study the semi-leptonic decay of $\mathrm{D}$ meson. In addition, we give the preliminary $\psi^{\prime \prime}$ resonance parameters with higher precision.

International Europhysics Conference on High Energy Physics

July 21st - 27th 2005

Lisboa, Portugal

${ }^{*}$ Speaker. 


\section{Introduction}

The study of $c \bar{c}$ quarkonium has made a significant progress since it has been found in 1974 . However, many problems and puzzles still remain unknown. The Beijing Spectrometer (BES) is a conventional solenoidal magnet detector that is described in detail in Ref. [1]. BESII is the upgraded version of the BES detector [2]. It is an important detector operated in charm energy region in the world. A lot of physics results have been published or reported at BES. We will present some recent BES results in this paper

\section{2. $J / \psi$ results}

\subsection{Observation of $\mathrm{X}(\mathbf{1 8 3 5})$}

Two years ago, we observed an enhancement near $p \bar{p}$ threshold in $J / \psi \rightarrow \gamma p \bar{p}$. BW fit gives $M=1859_{-10-25}^{+3} \mathrm{MeV} / \mathrm{c}^{2}, \Gamma<30 \mathrm{MeV} / \mathrm{c}^{2}$. According to BES measurement, we infer there is a large branching ratio of $X(1860) \rightarrow p \bar{p}$. Furthermore, the large branching ratio indicates the $\mathrm{X}(1860)$ has strong coupling to $p \bar{p}$. There are some theoretical predictions for the property of $X(1860)$. Recently, we observed a new resonance, $X(1835)$, via $J / \psi \rightarrow \gamma \pi^{+} \pi^{-} \eta^{\prime}$ [3]. It is likely to be a $p \bar{p}$ bound state since it dominantly decays to $p \bar{p}$ while its mass is above $p \bar{p}$ mass threshold.

\subsection{The $\sigma$ and $\kappa$}

There have been hot debates on the existence of $\sigma$ and $\kappa$. The decay of $J / \psi \rightarrow \omega \pi^{+} \pi^{-}$, with the $\omega$ decaying to $\pi^{+} \pi^{-} \pi^{0}$, is studied for $\sigma$ production [4]. Different parameterizations of BW are used in PWA(Partial Wave Analysis). The averaged pole position is

$$
(541 \pm 39)-i(252 \pm 42) M e V / c^{2}
$$

BES observed the $\kappa$ evidence in $J / \psi \rightarrow K^{*} K \pi$ decay channel [5], PWA gives its pole position

$$
841 \pm 30_{-73}^{+81}-i\left(309 \pm 45_{-72}^{+48}\right)
$$

\section{3. $\psi(2 S)$ results}

\subsection{Test of " $12 \%$ rule"}

From perturbative QCD, it is expected that both $J / \psi$ and $\psi(2 S)$ decaying into light hadrons are dominated by the annihilation of $c \bar{c}$ into three gluons or one virtual photon, with a width proportional to the square of the wave function at the origin, it is the so-called "12\%" rule,

$$
Q_{h}=\frac{\mathscr{B}_{\psi(2 S) \rightarrow h}}{\mathscr{B}_{J / \psi \rightarrow h}}=\frac{\mathscr{B}_{\psi(2 S) \rightarrow e^{+} e^{-}}}{\mathscr{B}_{J / \psi \rightarrow e^{+} e^{-}}} \approx 12 \%
$$

In order to test this rule, Many $\psi(2 S)$ decay channels have been investigated and their branching ratios are compared with the corresponding $J / \psi$ decay channels $[6,7,8]$. Table 1 lists some BES measured results for $\psi(2 S)$. One can find some channels follows this rule, some are enhanced and some are suppressed. 
Table 1: Branching ratios for the $\psi(2 S)$ and $J / \psi(\mathrm{PDG})$ decays.

\begin{tabular}{l|l|l|l|l}
\hline Mode & Channel & $\mathscr{B}(\psi(2 S))\left(\times 10^{-5}\right)$ & $\mathscr{B}(\mathrm{J} / \psi)\left(\times 10^{-4}\right)$ & $Q_{h}(\%)$ \\
\hline \multirow{7}{*}{ VP } & $\rho \pi$ & $5.1 \pm 0.7 \pm 1.1$ & $127 \pm 9$ & $0.40 \pm 0.11$ \\
\cline { 2 - 5 } & $K^{\star+} K^{-}+$c.c. & $2.9_{-1.7}^{+1.3} \pm 0.4$ & $50 \pm 4$ & $0.59_{-0.36}^{+0.27}$ \\
\cline { 2 - 5 } & $K^{\star 0} K^{0}+$ c.c. & $13.3_{-2.8}^{+2.4} \pm 1.7$ & $42 \pm 4$ & $3.2 \pm 0.8$ \\
\cline { 2 - 5 } & $\phi \pi^{0}$ & $<0.41$ & $<0.068$ & - \\
\cline { 2 - 5 } & $\phi \eta$ & $3.3 \pm 1.1 \pm 0.5$ & $6.5 \pm 0.7$ & $5.1 \pm 1.9$ \\
\cline { 2 - 5 } & $\phi \eta^{\prime}$ & $2.8 \pm 1.5 \pm 0.6$ & $3.3 \pm 0.4$ & $8.5 \pm 5.0$ \\
\cline { 2 - 5 } & $\omega \eta$ & $<3.2$ & $15.8 \pm 1.6$ & $<2.0$ \\
\cline { 2 - 5 } & $\omega \eta^{\prime}$ & $3.1_{-2.0}^{+2.4} \pm 0.7$ & $1.67 \pm 0.25$ & $19_{-13}^{+15}$ \\
\cline { 2 - 5 } & $\omega \pi^{0}$ & $1.87_{-0.62}^{+0.68} \pm 0.28$ & $4.2 \pm 0.6$ & $4.4_{-1.6}^{+1.8}$ \\
\cline { 2 - 5 } & $\rho \eta$ & $1.78_{-0.62}^{+0.67} \pm 0.17$ & $1.93 \pm 0.23$ & $9.2_{-3.3}^{+3.6}$ \\
\cline { 2 - 5 } & $\rho \eta^{\prime}$ & $1.87_{-1.11}^{+1.64} \pm 0.33$ & $1.05 \pm 0.18$ & $17.8_{-11.1}^{+15.9}$ \\
\hline
\end{tabular}

\subsection{Study of $\chi_{c J}$}

Based the $\chi_{c J}$ events from $\psi(2 S)$ sample via radiative decay of $\psi(2 S) \rightarrow \gamma \chi_{c J}$, many hadronic decays of $\chi_{C J}$ have been investigated. Table 2 lists some results. In addition, we measured the multipole amplitudes in radiative decay of $\psi(2 S) \rightarrow \gamma \chi_{c J}$ via $\psi(2 S) \rightarrow \gamma \chi_{c 0,2}, \chi_{c 0,2} \rightarrow \pi^{+} \pi^{-}, K^{+} K^{-}$[9]. The results are

$$
a_{2}^{\prime}=-0.051_{-0.036}^{+0.054}, \quad a_{3}^{\prime}=-0.027_{-0.029}^{+0.043}
$$

. The PWA of $\chi_{c 0} \rightarrow \pi^{+} \pi^{-} K^{+} K^{-}$was also performed [10]. The detailed analyses on $\chi_{c J} \rightarrow$

Table 2: BES results for $\chi_{c J}$ decay branching ratios $\left(\times 10^{-3}\right)$

\begin{tabular}{cccc}
\hline Decay channel & $\chi_{c 0}$ & $\chi_{c 1}$ & $\chi_{c 2}$ \\
\hline$K^{*}(892) K^{*}(892)$ & $1.78 \pm 0.34 \pm 0.34$ & $1.67 \pm 0.31 \pm 0.31$ & $4.85 \pm 0.56 \pm 0.88$ \\
$\omega \omega$ & $2.29 \pm 0.58 \pm 0.41$ & - & $1.77 \pm 0.47 \pm 0.36$ \\
$K_{S}^{0} K_{S}^{0}$ & $3.51 \pm 0.22 \pm 0.47$ & $<0.08(90 \%$ C.L. $)$ & $0.89 \pm 0.12 \pm 0.13$ \\
$K_{S}^{0} K_{S}^{0} \pi^{+} \pi^{-}$ & $6.5 \pm 0.6 \pm 1.0$ & $0.80 \pm 0.31 \pm 0.14$ & $3.24 \pm 0.61 \pm 0.55$ \\
$K_{S}^{0} K_{S}^{0} K^{+} K^{-}$ & $1.83 \pm 0.47 \pm 0.33$ & $0.31 \pm 0.19 \pm 0.06$ & $0.33 \pm 0.24 \pm 0.06$ \\
\hline
\end{tabular}

$K^{* 0}(892) \bar{K}^{* 0}(892), \omega \omega, K_{S} K_{s} h h$ are presented in Refs. [11, 12, 13]

\section{4. $\psi^{\prime \prime}$ results}

\subsection{Non- $D \bar{D}$ decay in $\psi^{\prime \prime}$}

BES first reported $\psi^{\prime \prime}$ non- $D \bar{D}$ decay in $\psi^{\prime \prime} \rightarrow \pi^{+} \pi^{-} J / \psi$, here $J / \psi$ is tagged by $\mu^{+} \mu^{-}$or $e^{+} e^{-}$pair. BES gives the measurement result, $\mathscr{B}\left(\psi^{\prime \prime} \rightarrow \pi^{+} \pi^{-} J / \psi\right)=(0.34 \pm 0.14 \pm 0.09) \%$ [14]. This result has been confirmed by CLEO via both $\psi^{\prime \prime} \rightarrow \pi^{+} \pi^{-} J / \psi$ and $\psi^{\prime \prime} \rightarrow \pi^{0} \pi^{0} J / \psi$.

\subsection{D meson exclusive semi-leptontic decay}

The branching ratio of $D^{0} \rightarrow K^{-} e^{+} v, \pi^{+} e^{0} v$ and $D^{+} \rightarrow K^{0} e^{+} v$ can be used to calculation CKM elements $\left|V_{c s}\right|$ and $\left|V_{c d}\right|$ and measure the form factor $\left|f_{+}^{K}(0)\right|$ and $\left|f_{+}^{\pi}(0)\right|$. In addition, the 
ratio $\frac{\Gamma\left(D^{0} \rightarrow K^{-} e^{+} v\right)}{\Gamma\left(D^{+} \rightarrow \bar{K}^{0} e^{+} v\right)}$ can be used to test isospin conservation. Table 3 lists some experimental results. With BES measured branching ratios, we obtain following results [15].

Table 3: Branching ratio for inclusive semi-leptonic decay(\%)

\begin{tabular}{|l|c|c|c|}
\hline \hline Experiment & $D^{0} \rightarrow K^{-} e^{+} v_{e}$ & $D^{0} \rightarrow \pi^{-} e^{+} v_{e}$ & $D^{+} \rightarrow \bar{K}^{0} e^{+} v_{e}$ \\
BES & $3.82 \pm 0.40 \pm 0.27$ & $0.33 \pm 0.13 \pm 0.03$ & $8.59 \pm 1.59 \pm 0.67$ \\
MARK-III & $3.4 \pm 0.5 \pm 0.4$ & $0.39_{-0.11}^{+0.23} \pm 0.04$ & $6.0_{-1.3}^{+2.2} \pm 0.7$ \\
PDG & $3.58 \pm 0.18$ & $0.36 \pm 0.06$ & $6.7 \pm 0.9$ \\
\hline
\end{tabular}

$$
\left|f_{+}^{K}(0)\right|=0.78 \pm 0.04 \pm 0.03, \quad\left|f_{+}^{\pi}(0)\right|=0.73 \pm 0.14 \pm 0.06, \quad\left|\frac{V_{c s}}{V_{c d}}\right|^{2}=0.043 \pm 0.01 \pm 0.003
$$

\subsection{Measurements of $\psi^{\prime \prime}$ and $\psi(2 S)$ resonant parameters}

Using hadronic cross-sections at 48 energy points from $3.65 \mathrm{GeV} / \mathrm{c}^{2}$ to $3.85 \mathrm{GeV} / \mathrm{c}^{2}$, we measure their resonance parameters of $\psi(2 S)$ and $\psi^{\prime \prime}$ by fitting $\psi(2 S)$ and $\psi^{\prime \prime}$ simultaneously. In such a way, the influence of $\psi(2 S)$ radiative tail to $\psi^{\prime \prime}$ has been accounted precisely. The preliminary results for $\psi^{\prime \prime}$ are

$$
M=3772.8 \pm 1.3 \mathrm{MeV} / \mathrm{c}^{2}, \Gamma_{t o t}=25.5 \pm 3.1 \mathrm{MeV} / \mathrm{c}^{2}, \Gamma_{e^{+} e^{-}}=224 \pm 30 \mathrm{eV} / \mathrm{c}^{2}
$$

\section{References}

[1] J. Z. Bai et al., BES Collab., Nucl. Instr. Meth. A344, 319 (1994).

[2] BES Collaboration, Nucl. Instr. Meth., A344 319 (1994), A458 627 (2001).

[3] BES Collaboration, M. Ablikim, et al., accepted by Phys. Rev. Lett. hep-ex/0508025

[4] BES Collaboration, M. Ablikim, et al., Phys. Lett. B598 149 (2005).

[5] BES Collaboration, M. Ablikim, et al., hep-ex/0506055.

[6] BES Collaboration, M. Ablikim, et al.,Phys. Lett. B614, 37 (2005)

[7] BES Collaboration, M. Ablikim, et al., Phys. Lett. B619, 247(2005)

[8] BES Collaboration, M. Ablikim, et al., Phys. Rev. D70, 112007(2004)

[9] BES Collaboration, M. Ablikim, et al., Phys. Rev. D70, 092004 (2004).

[10] BES Collaboration, M. Ablikim, et al., Phys. Rev. D72, 092002 (2004).

[11] BES Collaboration, M. Ablikim, et al., Phys. Rev. D70, 092003 (2004).

[12] BES Collaboration, M. Ablikim, et al., Phys. Lett. B630, 7 (2005).

[13] BES Collaboration, M. Ablikim, et al., Phys. Lett. B630, 21 (2005).

[14] BES Collaboration, M. Ablikim, et al., Phys. Lett. B605, 63 (2005).

[15] BES Collaboration, M. Ablikim, et al., Phys. Lett. B608, 24 (2005). 